\title{
SIMULATION-BASED EVALUATION OF LIGHT POSTS AND STREET SIGNS AS 3-D GEOLOCATION TARGETS IN SAR IMAGES
}

\author{
S. Auer ${ }^{\mathrm{a}}, \mathrm{U} \cdot$ Balss $^{\mathrm{a}}$ \\ ${ }^{a}$ Remote Sensing Technology Institute, German Aerospace Center (DLR), 82234 Wessling-Oberpfaffenhofen, Germany - \\ (stefan.auer, ulrich.balss)@dlr.de
}

KEY WORDS: Synthetic Aperture Radar (SAR), Ground Control Point (GCP), StereoSAR, interpretation, street objects, simulation

\begin{abstract}
:
The assignment of phase center positions (in 2D or 3D) derived from SAR data to physical object is challenging for many man-made structures such as buildings or bridges. In contrast, light poles and traffic signs are promising targets for tasks based on 3-D geolocation as they often show a prominent and spatially isolated appearance. For a detailed understanding of the nature of both targets, this paper presents results of a dedicated simulation case study, which is based on ray tracing methods (simulator RaySAR). For the first time, the appearance of the targets is analyzed in 2D (image plane) and 3D space (world coordinates of scene model) and reflecting surfaces are identified for related dominant image pixels. The case studies confirms the crucial impact of spatial resolution in the context of light poles and traffic signs and the appropriateness of light poles as target for 3-D geolocation in case of horizontal ground surfaces beneath.
\end{abstract}

\section{INTRODUCTION}

Up to now, the prevalent application areas of spaceborne Synthetic Aperture Radar (SAR) are weather and time of day independent observations of the earth surface. However, several recent studies (Schubert et al., 2011) (Eineder et al., 2011) (Schubert et al., 2012) (Balss et al., 2014) revealed that SAR offers as well the ability for high precision measurement of absolute positions. In particular, localization accuracy of corner reflectors at the centimeter level is proven for the German SAR satellites TerraSAR-X and TanDEM-X (Balss et al., 2014). This progress in localization accuracy triggers new application scenarios for SAR (e. g. (Runge et al., 2016)) in areas like StereoSAR, geodetic SAR or absolute ranging. However, the benefit of knowing the absolute phase center position of a point scatterer is heavily dependent on the assignability of the measured position to a real point on a real object. Here, numerical simulations are a valuable instrument to bridge this gap.

\section{MOTIVATION AND SCOPE}

There is a wide variety of measurement methods for accurate 3-D capture of the environs which can deliver highly accurate relative coordinates but are in need of so-called Ground Control Points (GCPs) with known absolute 3-D coordinates when their results shall be geolocalized with respect to a global reference frame. A frequently used mean to capture precise absolute 3-D coordinates of GCPs are in situ measurements based on the Global Navigation Satellite System (GNSS). But there are many situations where a terrestrial geodetic survey is not practical or even impossible: The journey for the staff may be too costly or the region inaccessible or too dangerous. As soon as a multitude of GCPs is required, in situ survey gets above all very laborious and time consuming.

Therefore, (Balss et al., 2016) proposed a StereoSAR (Chen and Dowman, 2001) (Sansosti, 2004) (Gisinger et al., 2015) (Fratarcangeli et al., 2016) based remote sensing method to efficiently gather entire networks of 3-D points, to be exploited as GCPs for other sensors like e. g. laser scanners (LiDAR) or stereo cameras, without the necessity to enter the region. Prerequisite for

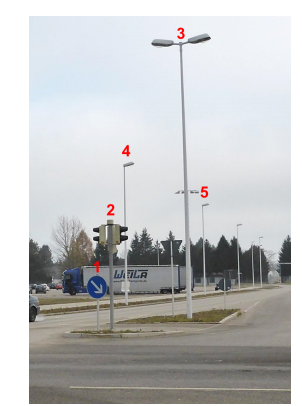

(C) Vermessungsbuero Patrzek 2015

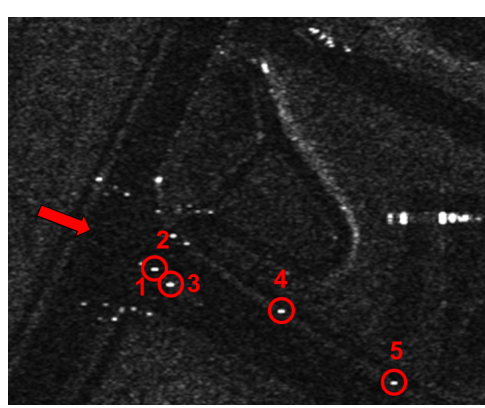

(c) DLR 2014
Figure 1. On-site inspection vs. spaceborne SAR; left: photo of a road junction in Oberpfaffenhofen (Germany); right: close-up of this junction in a TerraSAR-X High Resolution Spotlight (HS300) image; a red arrow marks the viewing direction of the optical image. Some prominent posts (labels 1-5) are likewise identifiable in photo and SAR image.

the extraction of 3-D points by this method and for their transfer as GCPs on the target data is an unambiguous identification of the 3-D points in at least two SAR images acquired from significantly different viewing angle (for extraction) and in the target data (for transfer).

In particular in urban areas, SAR images acquired by state-of-theart high resolution SAR sensors like TerraSAR-X or COSMOSkyMed show a myriad of point-like objects which are however of different value for the exploitation as GCPs for other sensors. The majority of them stem from building structures like protrusions or window cutouts. Often, such structures have complex radar reflection properties and it is hard to assign their phase center position to an actual, single object (Gernhardt et al., 2014) (Auer and Gernhardt, 2014). In contrast, poles of lamps or traffic signs show rather manageable reflection properties. In particular, corresponding observations of sole free-standing lamp poles or traffic signs are easily assignable because they cannot be confused with nearby similar objects. Many of them can be found in urban areas and along of roads and motorways (as it is illustrated by the example in Fig. 1). The attainable localization accuracy 

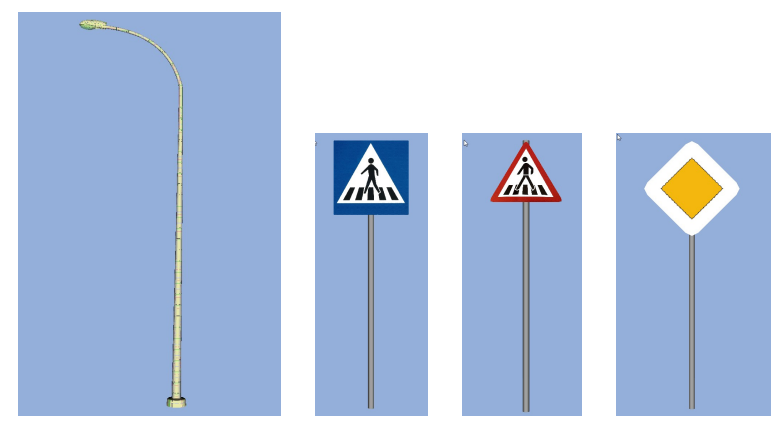

Figure 2. 3D models for SAR simulation.

depends on the radar brightness on the point scatterer relative to its vicinity. As analyzed in (Balss et al., 2016) by cross-check with GNSS reference coordinates, 3-D location accuracy of about $10 \mathrm{~cm}$ for lamp poles and less than $30 \mathrm{~cm}$ for poles of traffic signs is obtained on base of TerraSAR-X data take.

The optimum would be a sole straight metallic pole without any additions and perpendicularly mounted on a flat asphaltic road surface, as this configuration constitutes a radar reflector which occurs as ideally focused point in a SAR image. There is a double bounce between the front edge of the pole and the ground. The phase center of the radar echo, which yields the 3-D point, corresponds to the base point of the pole.

However, real poles along roads somewhat differ from this idealized model: The poles carry lamp housings or traffic signs; and a curved design is typical for a frequent type of modern lamp posts (see Fig. 2). Real roads are almost always convex and usually declined for drain rain water. If there is a sidewalk along the road, poles are often mounted near the edge of the sidewalk, face away from the road, and the curb constitutes a step between the reflecting surfaces of sidewalk and road. Any of these effects may cause a small offset between the base point of the pole (which corresponds to the phase center of the bare pole on a flat road surface) and the true phase center position in the real world scenario. Thus, understanding the underlying backscatter geometry and evaluating the expected amount of a potential offset between phase center and base point improves the interpretability of the StereoSAR based 3-D points.

Due to the complex shapes of real pole (with mounted additions) and non-flat road surface, numerical simulations are the most appropriate mean to investigate the overall effect on the radar signature of the scatterer and to localize the resulting radar phase center relative to the base point of the pole.

This paper presents results of a simulation case study dedicated to light poles and traffic signs in order to better understand the inherent information in the context of high-resolution SAR images. The simulation method and the case study scenario is described in the following section.

\section{INTRODUCTION OF RAYSAR}

RaySAR is a 3D SAR simulator (Auer, 2011) developed in a cooperation between DLR and TU Munich (open access on GitHub: see (Auer et al., 2016)). It incorporates an adapted version of the POV-Ray ray tracer (POV-Ray, 2017) and MATLAB tools to generate SAR image layers and analyze the nature of selected scene objects. Besides imagery in the azimuth-range plane (SAR image, reflectivity maps, separate layers for reflection levels), 3D
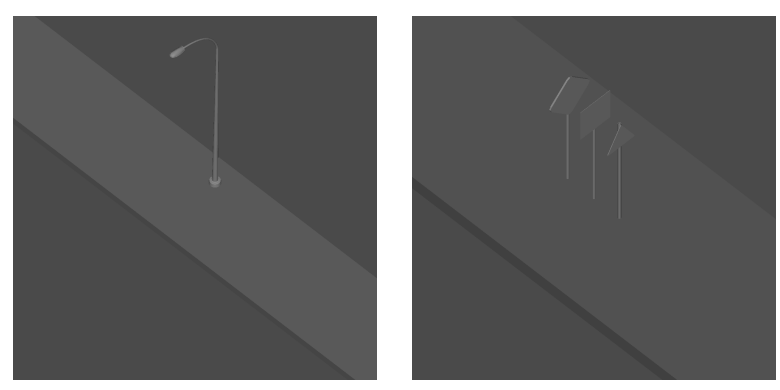

Figure 3. Rendered images of scenario 1 (light pole; left) and scenario 2 (street signs; right) for aspect angle $45^{\circ}$.

positions of signal phase centers and the extent of signal reflecting surfaces can be analyzed. To this end, 3D information is projected into the scene model scene where it is merged with the 3D shape of objects. For the case study below, two object models are under consideration: a curved light pole and standard street signs. In particular, the impact of the following aspects is of interest:

- Post conicity (i.e. diameter change with height)

- Impact of specular and diffuse reflections

- Impact of object curvature (light pole) and surface slopes (road surface)

- Resolution effects for nearby street signs

- Position of prominent signal phase centers

\section{SCENARIOS}

Two simulation scenarios are defined for covering the aspects of interest above. Scenario 1 concentrates on the imaging of light poles.

- Scene model: curved light pole on pavement, distance between light pole center and pavement border: $1.5 \mathrm{~m}$, road sloped across the heading direction by $3.43^{\circ}(6 \%), 0^{\circ}$ along the road.

- Focus: Simulation of SAR images for different sensor perspectives; analysis of signal phase center positions.

- Simulation: change of aspect angle: $0^{\circ}$ to $45^{\circ}$, signal incidence angle: $40^{\circ}$; spatial resolution of SAR image: TerraSARX Spotlight, TerraSAR-X Staring Spotlight.

Scenario 2 is focused on groups of traffic signs:

- Scene model: group of street signs in a row (approx. $6 \mathrm{~cm}$ diameter; spatial distance of $0.5 \mathrm{~m}$ between the street signs); pavement and road modeling equal to scenario 1 . The spatial distance between the signs has been selected near the resolution limit of TerraSAR-X.

- Focus: Impact of sensor resolution on the traffic sign appearance.

- Simulation: change of aspect angle: $0^{\circ}$ to $45^{\circ}$; signal incidence angle: $40^{\circ}, 49^{\circ}, 58^{\circ}$; spatial resolution: TerraSAR-X HR Spotlight, TerraSAR-X Staring Spotlight. 
Appropriate object models have been procured (curved light pole with 8099 polygons, priority sign with 4757 polygons, pedestrian crossing sign with 4781 polygons, pedestrian crossing warning sign with 5316 polygons; see Fig. 2), representing the basic geometric rules pertinent to the targets. The 3D models provide the necessary level of geometric detail as well as the intended properties (e.g. decrease of light pole diameter, curvature of surface, geometric description of lamp head details). Fig. 3 provides a visual impression of the defined scenarios. The density of rays (for ray tracing) is $1.5 \mathrm{~cm}$ at the light pole and $0.25 \mathrm{~cm}$ at the street signs.

The following parameters have been used for generating the SAR images:

- High resolution spotlight mode: spatial resolution $1.10 \mathrm{~m}$ x $0.59 \mathrm{~m}$ (azimuth, range), pixel spacing $0.87 \mathrm{~m} \mathrm{x} 0.46 \mathrm{~m}$ (azimuth, range)

- Staring spotlight mode: spatial resolution $0.23 \mathrm{~m} \times 0.59 \mathrm{~m}$ (azimuth, range), pixel spacing $0.17 \mathrm{~m}$ x $0.46 \mathrm{~m}$ (azimuth, range)

- Signal integration: coherent summation of signal contributions for each image pixel

In the remainder of the paper, the high resolution spotlight mode of TerraSAR-X is referred to as "spotlight mode" (other mode: staring spotlight mode).

\section{SIMULATION FOR LIGHT POLE SCENARIO}

\subsection{Simulation of Images}

The simulation case study starts with scenario 1 (light pole). As a first step, the spatial distribution of signal contributions is analyzed for varying aspect angles (see Fig. 4). It is seen that the allover appearance of the light pole remains comparable. Local double (and occasional triple reflections) from the lamp head (near range) are followed by an interval of direct backscattering (pole, ground). At the bottom end of the pole, a mix of double reflections (spatially distributed) and point-like triple reflection phase centers with varying positions occur. Finally, higher-order reflection levels end up in far range, again with varying positions for different aspect angles. Ground parts are represented by diffuse signal response (direct backscattering). The height step at the pavement border leads to a linear arrangement of double reflection phase centers (green line) for aspect angles bigger than $0^{\circ}$.

Fig. 4 shows only the geometric part. Accordingly, it visualizes the geometric composition of signal components in the scene. A look to simulated SAR images based on the signal distribution map adds the radiometric aspect. To this end, the discrete point cloud (signal positions in azimuth, range, elevation) is imposed with a image grid in azimuth and range. Thereafter, the signal intensities are summed coherently for each image pixel.

Fig. 5 presents the simulated imagery with the image specifications for TerraSAR-X spotlight mode. It can be seen that the setting of surface parameters (low roughness for surfaces, strong specular behavior for metallic surfaces of the light pole) leads to one dominant point signature, which has a stable position, and a linear signature with varying intensity level for different aspects.
Considering Fig. 4 and Fig. 5, the point signature can be understood as a spatial integration of double reflection signals (signal path: antenna - ground - post - sensor or antenna - post - ground - sensor).

The intensity of the point signature varies with aspect angle. This can be seen from the simulated images. Each image is scaled to 8 bit gray values considering the maximum and minimum intensity in the scene. Hence, the variation of the (light pole signature) intensity maximum leads to an occasional emphasis of the (ground) intensity. Due to the same reason, the intensity of the double reflection line signature varies (stronger appearance for aspect angles $15^{\circ}, 25^{\circ}, 35^{\circ}$, and $45^{\circ}$ ). The reason is the impact of the light pole geometry leading to discontinuities for changing perspectives (e.g. pole curvature described with flat polygons).

Also the shape of the point signature appears to change slightly for different aspect angles. To analyze this further, SAR images with higher spatial resolution are simulated. Fig. 6 provides the overview of simulated images with the specifications for TerraSAR-X staring spotlight mode. It is obvious that the shape of the point signature noticeably changes with aspect angle as smearing effects occur in azimuth direction. Likely, the standard double reflection of type ground - pole is mixed with double reflections related to the curved part of the light pole (including lamp head) and the ground.

Interestingly, dominant signatures occur despite the $6 \%$-slope imposed on the road. This may serve as an indicator that signal contributions related to the road surface are not dominant ones.

\subsection{D Analysis}

In this section, the nature of signal phase centers is analyzed for scenario 1 (light pole on pavement). At the beginning, the focus is on the visual impression of simulation results. To this end, one aspect is selected (aspect angle: $45^{\circ}$, signal incidence angle: $40^{\circ}$ ) and the related signal phase centers and relevant ray-surface intersection points are projected back into the 3D model space of the light pole scene. Thereafter, position changes are analyzed for varying aspect angles in order to characterize the perspectiverelated behavior of prominent phase centers.

RaySAR allows for the interactive selection of pixels of interest in order to generate height profiles and write ray-surface intersection points to a file. Besides, the 3D positions of selected reflection levels (here: signal double reflection) can be projected into the light pole model scene. Results are presented below.

5.2.1 Height Profiles The pixel selection (brightest one) in the SAR image shown in Fig. 5j defines two cross-sections over the image (one in range, one in azimuth) which intersect at the pixel center. The width of the section (perpendicular to the slice direction) is selected as being equal to the TerraSAR-X pixel spacing in azimuth and range, respectively. All signal phase centers within the section are collected, color-coded based on the reflection level, and visualized in a plot. In this context, the elevation information (perpendicular to the azimuth and range axes) can be visualized in true elevation (across azimuth and range) or height over ground (relative height with respect to minimum single reflection contribution in the scene).

Fig. 7a shows the visualization of elevation values along the cross-section pointing in range direction. Direct responses are distinguishable for the light pole as well as for ground parts (road 


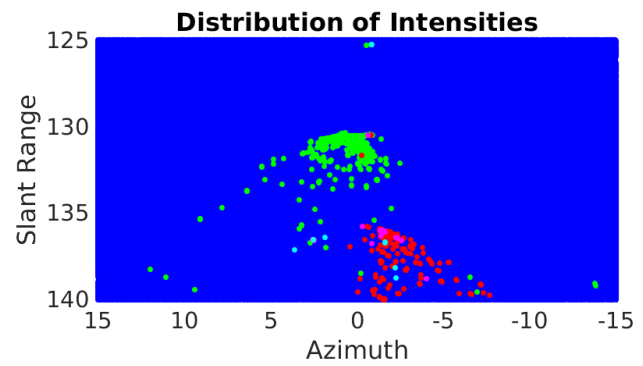

(a) Aspect angle: $0^{\circ}$

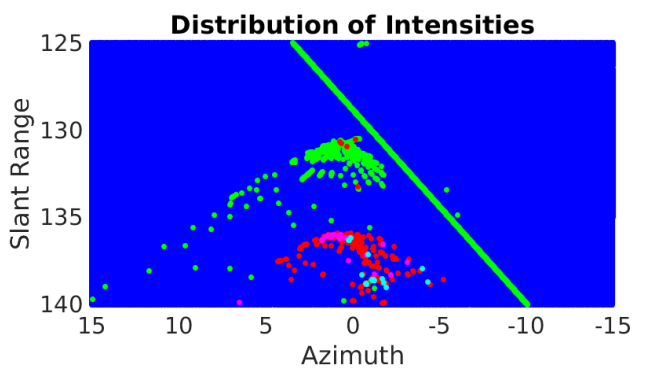

(c) Aspect angle: $30^{\circ}$

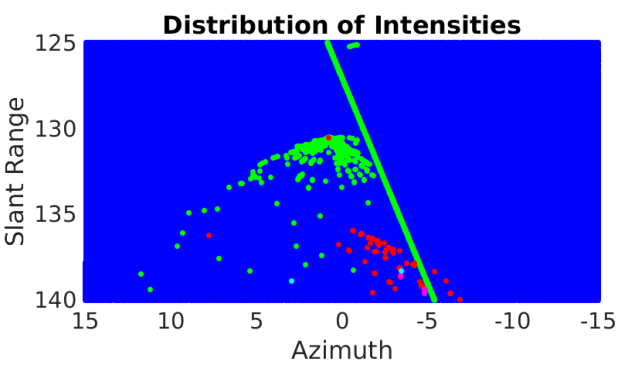

(b) Aspect angle: $15^{\circ}$

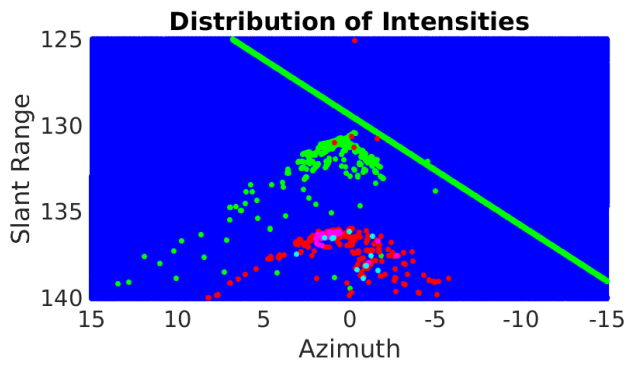

(d) Aspect angle: $45^{\circ}$

Figure 4. Simulated phase center positions for light pole in azimuth and range; blue: single reflection, green: double reflection, red: triple reflection, magenta: fourfold reflection, cyan: fivefold reflection, white: shadow. Unit on axes: meter.

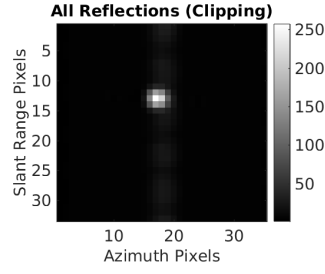

(a) Aspect angle: $0^{\circ}$

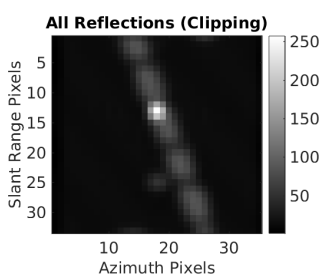

(f) Aspect angle: $25^{\circ}$

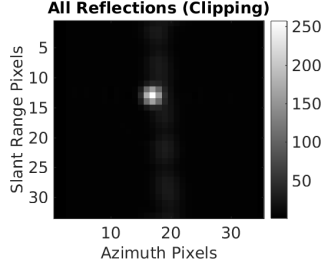

(b) Aspect angle: $5^{\circ}$

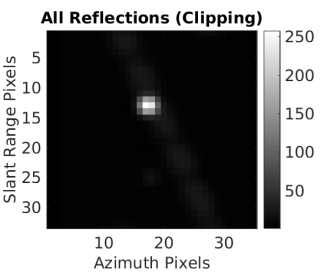

(g) Aspect angle: $30^{\circ}$

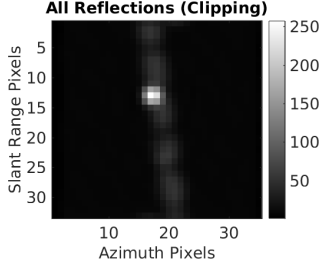

(c) Aspect angle: $10^{\circ}$

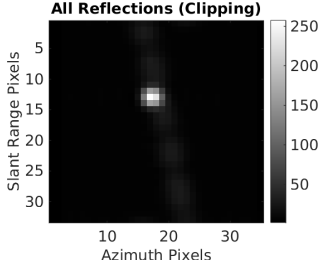

(d) Aspect angle: $15^{\circ}$

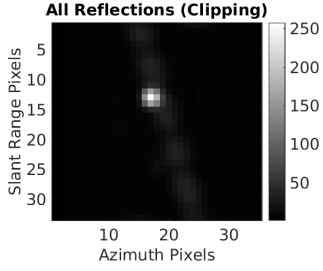

(e) Aspect angle: $20^{\circ}$

Figure 5. Simulated SAR images for light pole (spotlight mode).

and pavement). Besides, many double reflection phase centers are seen. On the left end, a linear arrangement of double reflection phase centers is related to the corner line between the road and the pavement. Double reflection phase centers near the lower end of the light pole are linked to the signal path "antenna - light pole - ground - sensor" or "antenna - ground - light pole - sensor" and form a cluster within a elevation interval of approx. 0.6 $\mathrm{m}$. Phase centers of high order (triple to fivefold) relate to signal multiple reflections at light pole parts and the ground and, hence, reveal bigger range values. Phase centers with the same slant range value refer to the same SAR image pixel.

Fig. $7 \mathrm{~b}$ shows phase center elevation values transformed into heights over ground. Here, the double reflection phase center positions are better comparable to the road level (sloped) and the pavement level (horizontal). It is seen that signal multiple reflections in the scene end up in phase centers approx. $5 \mathrm{~m}$ below the ground level, whereas double reflections mostly remain on the height level of the pavement. Phase center clusters occur at light pole height steps (signal path: ground - light pole base; light pole base - pole) as well as on the pavement surface behind the light pole (origin: signal double reflections including diffuse signal responses from the pavement behind the light pole). Double reflection phase centers below the pavement level occur due to the signal interaction with light pole components and the sloped road.

The simulated height profiles indicate that a visual identification of one dominant phase center (as expected from the simulated SAR image) is not possible. To clarify this, a dedicated analysis on the behavior of cluster centers in the scene is conducted in section 5.2.3.

5.2.2 Projections to Model Space Fig. 8 shows the result of geometric projections into the 3D model space which are con- 


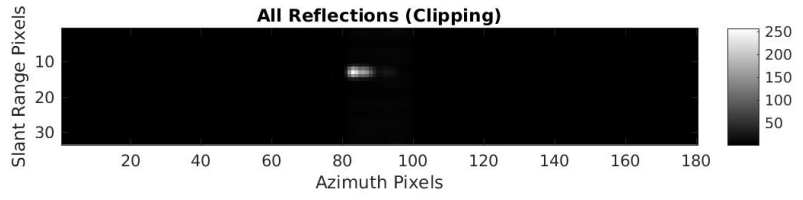

(a) Aspect angle: $0^{\circ}$

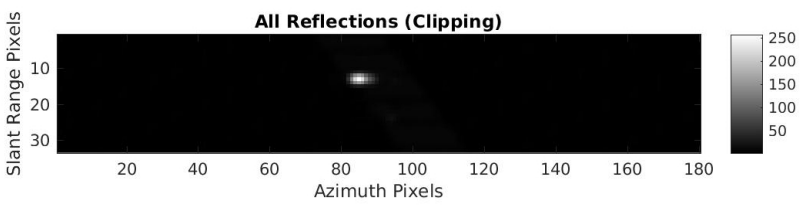

(c) Aspect angle: $10^{\circ}$

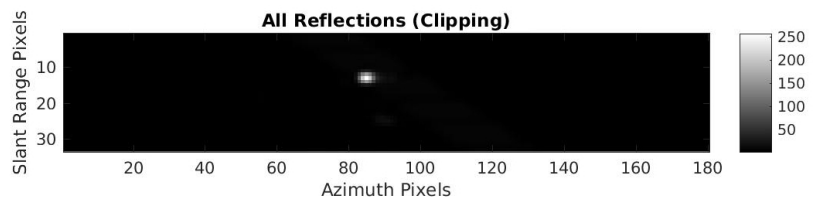

(e) Aspect angle: $20^{\circ}$

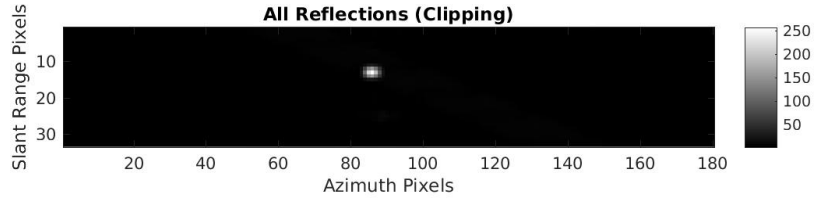

(g) Aspect angle: $30^{\circ}$

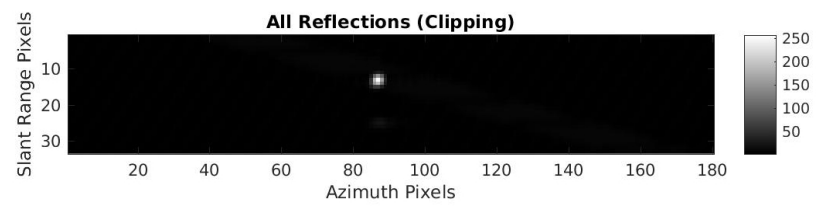

(i) Aspect angle: $40^{\circ}$

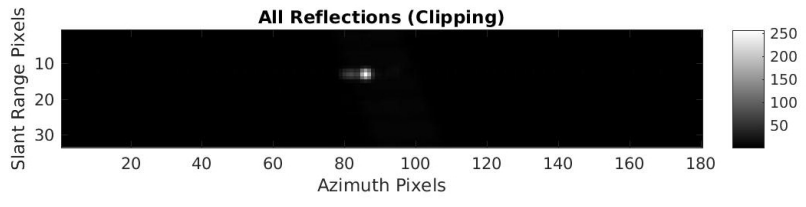

(b) Aspect angle: $5^{\circ}$

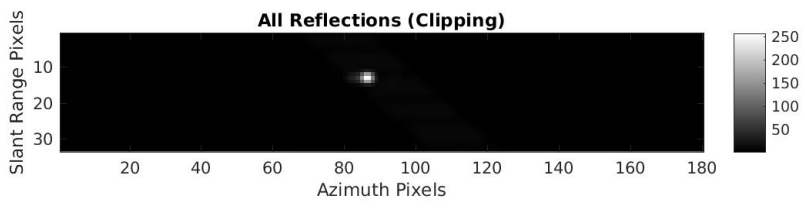

(d) Aspect angle: $15^{\circ}$

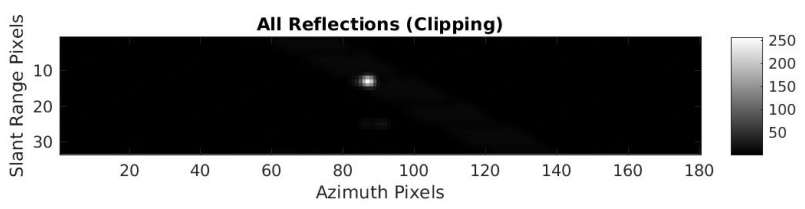

(f) Aspect angle: $25^{\circ}$

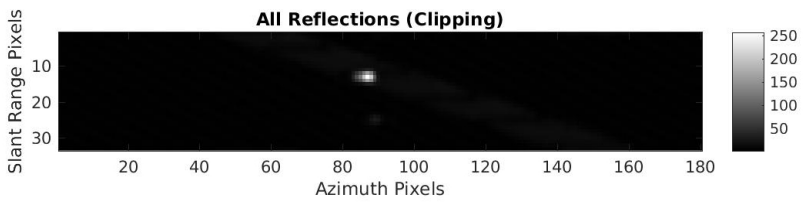

(h) Aspect angle: $35^{\circ}$

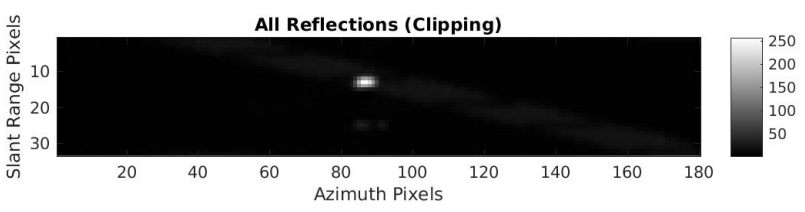

(j) Aspect angle: $45^{\circ}$

Figure 6. Simulated SAR images for light pole (staring spotlight mode).

ducted based on the given SAR sensor perspective, the center of the 3D model scene and the simulated phase center distances derived from ray tracing. The phase center models (cube with $3 \mathrm{~cm}$ side length) have been exported into the Wavefront format (.obj). Besides, the 3D coordinates are stored to a text file (required for analysis shown in Section 5.2.3).

In the context of signal phase centers (see Fig. 8a), the focus is on signal double reflections (other reflection levels contribute too little intensity to the simulated SAR image to be relevant). Besides the linear arrangement of phase centers (intersection line of road and pavement), different types of double reflection phase centers occur at the surrounding of the light pole. The majority of phase centers are concentrated at the bottom end of the light pole. The surface behind the light pole is covered by phase centers arranged along lines, which are related to signal interaction with the light pole (first signal bounce of specular type) and the ground behind the light pole (second signal bounce of diffuse type). The linear arrangement of the phase centers occurs due to to the representation of the light pole by means of flat polygons and the discrete scene sampling of the ray tracer (for curved light poles the distribution of phase centers is expected to be continuous). In the SAR image, the corresponding image signatures are expected to be of weak intensity and smeared (lack of stability of the reflection effect along the synthetic aperture).

Fig. $8 \mathrm{~b}$ visualizes all scene surfaces contributing to the most prominent pixel in the SAR image (compare Fig. 5j). To this end, all ray-polygon intersection points contributing to the pixel have been selected and exported to a Wavefront model file (cube size: $3 \mathrm{~cm}$ ). Surfaces with direct signal response are marked in blue color (pavement, parts of the light pole surface), surfaces contributing to signal double reflections are marked in green color. It is seen again that ground and light pole parts contribute to the prominent pixel.

5.2.3 Position of Dominant Phase Centers Fig. 8a visualizes all phase centers related to signal double reflections. However, only some of them are expected to be of prominent appearance (merge of many signal double reflections at a specific position). This assumption is also supported by the simulated SAR images of the lamp scene (see Figs. 5 and 6), where one salient point signature is obtained for varying sensor perspectives. In this section, prominent phase centers are analyzed for their appearance and position.

RaySAR can be used to transform signal phase centers to their 


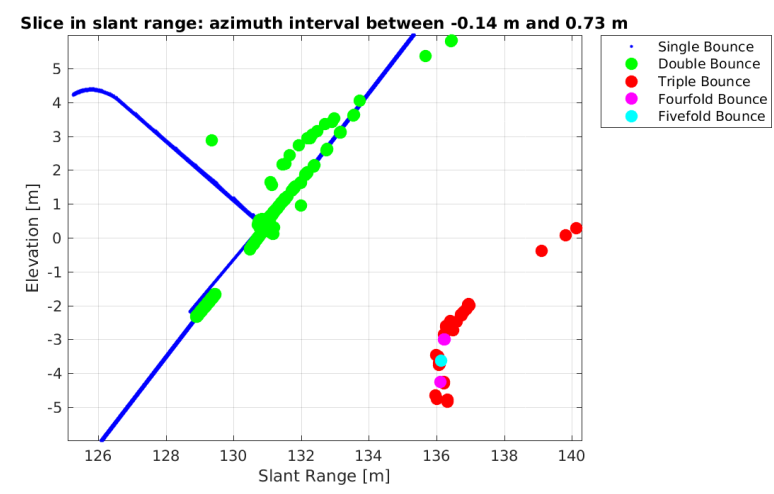

(a) Elevation values (across the line-of-sight)

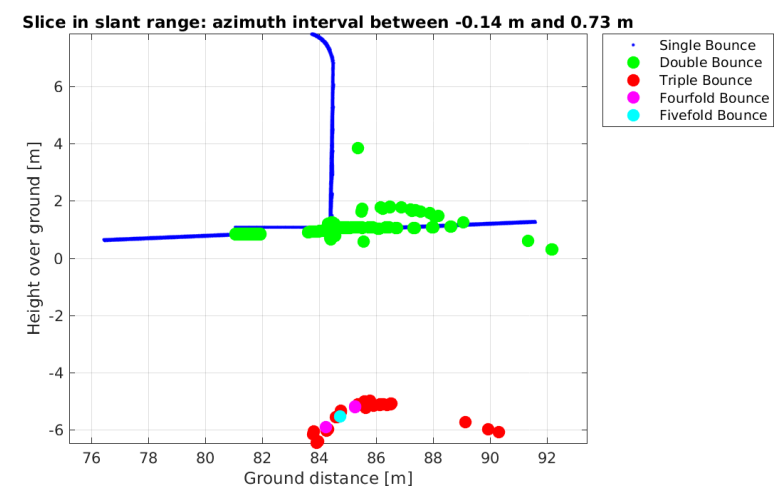

(b) Heights over minimum ground level in the scene. Horizontal pavement, sloped road surface.

Figure 7. Simulated height profiles in range direction based on the selection of the most prominent pixel in the SAR image. The azimuth interval refers to the pixel size (= TerraSAR-X pixel spacing for spotlight mode).

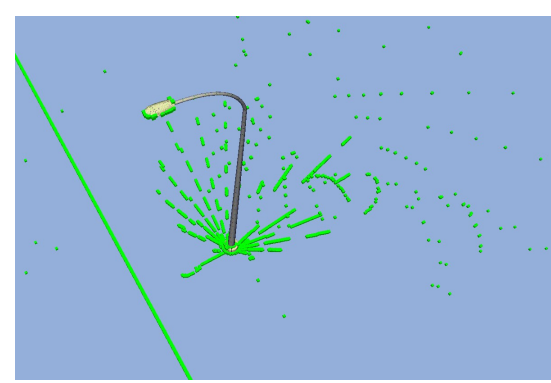

(a) Phase centers related to signal double reflections, concentrated at the lower end of the post or spatially distributed at the ground surrounding the post (phase centers represented by cubes of size $3 \mathrm{~cm}$ ). The linear arrangement of phase centers marks the pavement corner line.

Figure 8. Projection of phase centers and reflecting surfaces into the light pole 3D model.

positions in the model world coordinate system. In this context, the world coordinates are stored in a text file in combination with the number of occurrence, which is derived by counting the simulated 3D phase centers in a local neighborhood. In case of the lamp model example, the neighborhood threshold has been set to $1 \mathrm{~cm}$ in 3D space. Based on that, dominant phase centers with an occurrence number above a defined threshold are combined for the aspect angles between $0^{\circ}$ and $45^{\circ}$ and analyzed for their spatial spread.

In total, 204k double reflection phase centers occur for all 10 as-
Table 1. Summary of phase center geometry and aspect dependence (values rounded to $\mathrm{cm}$ ). Compare: position of light pole base point at $(0.78,0,0)$. Maximum occurrence level: 174 (= number of merged signal contributions at one point). $\mathrm{dx}, \mathrm{dy}, \mathrm{dz}$ $=$ intervals on world coordinate axes including all phase center

positions, Asp. = Number of supported aspect angles.

\begin{tabular}{|c|c|c|c|c|c|c|c|}
\hline Level & $\mathrm{x}$ & $\mathrm{y}$ & $\mathrm{z}$ & $\mathrm{dx}$ & $\mathrm{dy}$ & $\mathrm{dz}$ & Asp. \\
\hline 10 & 0.79 & -0.05 & 0.00 & 0.49 & 0.16 & 0.08 & 10 \\
\hline 50 & 0.84 & -0.06 & 0.00 & 0.10 & 0.04 & 0.00 & 10 \\
\hline 100 & 0.85 & -0.05 & 0.00 & 0.05 & 0.03 & 0.00 & 7 \\
\hline
\end{tabular}

pects (from $0^{\circ}$ to $45^{\circ}$ with step width $5^{\circ}$ ). Setting the occurrence threshold to 50 yields a spatial concentration of phase centers (see top of Fig. 9). Still, phase centers are contributed from all aspects (see last column Table 1). Using the threshold 100, only the most prominent phase centers remain (bottom of Fig. 9), which further stabilizes the position information but leads to no entry for 3 aspect angles. The height level of the group (at the light pole base point) indicates that the pavement contributes the second surface for the signal double reflection. The mean position of the phase center group is shifted by $8 \mathrm{~cm}$ with respect to the light pole base point. This can be explained with signal reflections occurring on the surface of the light pole (diameter varies with height; approx. $8 \mathrm{~cm}$ at the bottom end of the light pole). The tilted road surface does not trigger dominant phase centers.

The simulation result does not serve as a proof for light poles being persistent targets. Real scenes are made up of many more objects and characterized by a strong variation of surface properties. However, the simulation confirms for the representative scene set-up that dominant phase centers remain within a spatially restricted region for varying aspects. This also contributes to the understanding of one salient point signature in the simulated SAR image obtained for all aspect angles. Due to the geometry of the light pole surface, the phase center position cannot remain the same for different aspect angles (nonzero diameter).

\section{SIMULATION FOR STREET SIGN SCENARIO}

Simulation scenario 2 is concerned with 3 street signs positioned at the equal pavement-road scene with a spatial distance of $0.5 \mathrm{~m}$ in between them. The focus of the simulation is on the impact of the sensor resolution on the sign group appearance. Related effects are analyzed for changing aspect angles $\left(0^{\circ}, 15^{\circ}, 30^{\circ}, 45^{\circ}\right)$ and a signal incidence angle of $40^{\circ}$. Again, the analysis starts with a view onto the spatial distribution of signal contributions and continues with the visual perception of the simulated SAR images.

Fig. 10 shows the signal distribution for the different aspect angles. Imaging effects related to the ground and pavement height step are equal to the light pole example discussed above. Differently, however, the mixture of signal contributions in the center part of the image is now related to the group of traffic signs. From the geometric viewpoint, it is impossible to assign signal components to the individual signs. Only the shapes of the shadow areas (white color) indicate the presence of three scene objects. This impression is confirmed by the simulated SAR images (see Fig. 11), which have been generated under the assumption of TerraSAR-X spotlight mode. For the aspect angle of $15^{\circ}$, one point signature is obtained, which appears to be slightly smeared in range direction. For bigger aspect angles, the geometric arrangement of the street signs leads to a rotated (for $30^{\circ}$ ) and disturbed (for $45^{\circ}$ ) point signature. Under the assumption of staring 

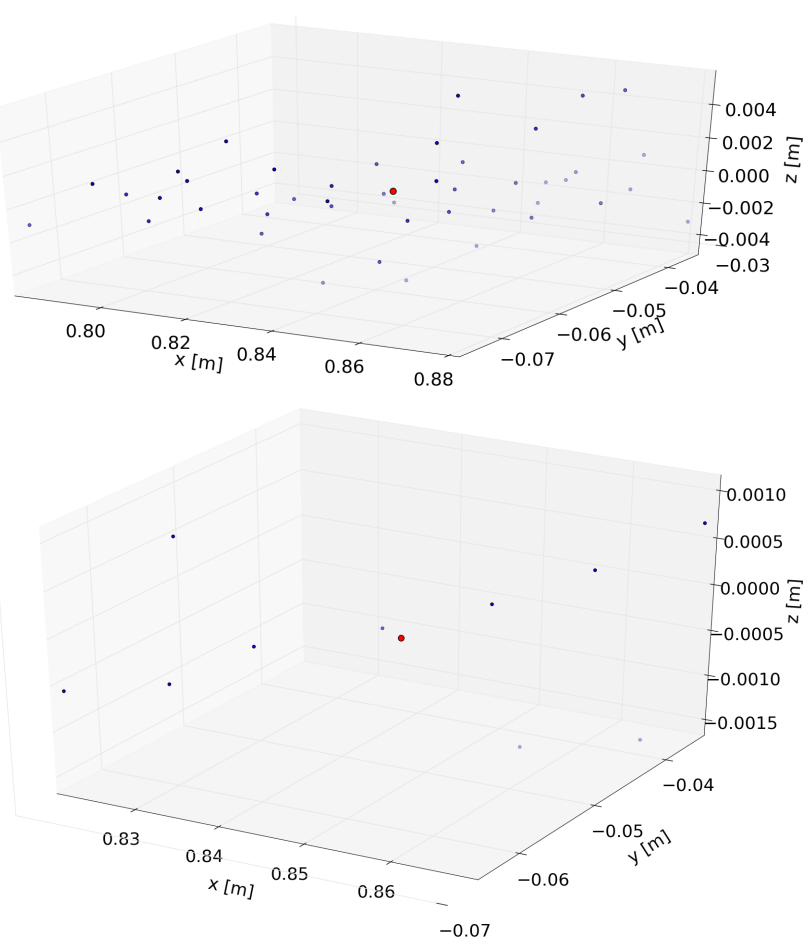

Figure 9. Signal double reflection phase centers for occurrence thresholds 50 (contributions from all aspects) and 100 (contributions from 7 aspects).

spotlight mode, these effects become better distinguishable (see Fig. 12). Starting with a dot-like shape for aspect $15^{\circ}$, the signature is stretched and rotated with an increase of the aspect angle.

\section{ADDITIONAL REMARKS}

At this point, some additional comments are due:

- Height steps: Height steps participating at signal multiple reflections influence the height level of the phase centers. For the light pole example in the case study, however, dominant phase centers were always related to the pavement surrounding the light pole. Therefore, the phase center height was equal to the height of the light pole base point.

- Lamp heads: The simulation confirms diffuse signal reflections for the light pole example (no specular ones). However, the corresponding signal strength is weak compared to the dominant signal double reflection of type "light pole ground". Hence, it is expected that prominent lamp head responses require specular direct response or triple reflections. However, this effect is not general for different aspects and, hence, is not stable for varying aspect angles.

- Signal polarization: RaySAR does not cover the simulation of signal polarization. Accordingly, assumptions in the context of the study are only based on theory. Considering the first signal interaction with the scene, the difference between horizontal and vertical polarization is expected to be small for metallic light poles (weak impact of Brewster angle in vertical polarization) and prominent for horizontal non-metallic surfaces (remarkable impact of Brewster angle for vertical polarization at mid-incidence angles). Accordingly, as the dominant phase centers are related to reflections including the horizontal pavement, light pole phase centers

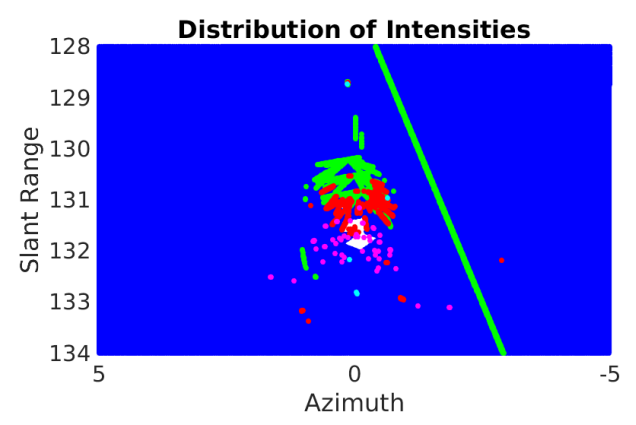

(a) Aspect angle: $15^{\circ}$

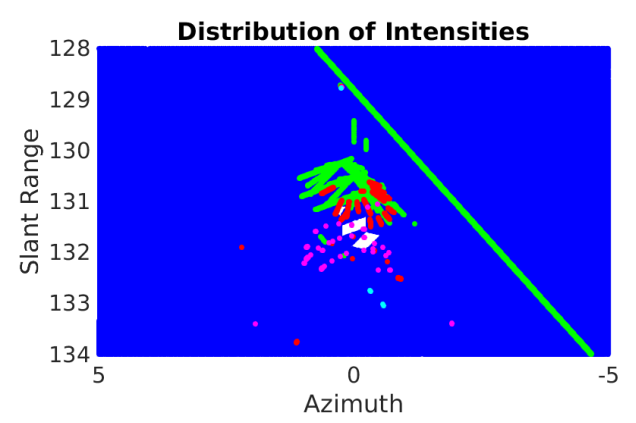

(b) Aspect angle: $30^{\circ}$

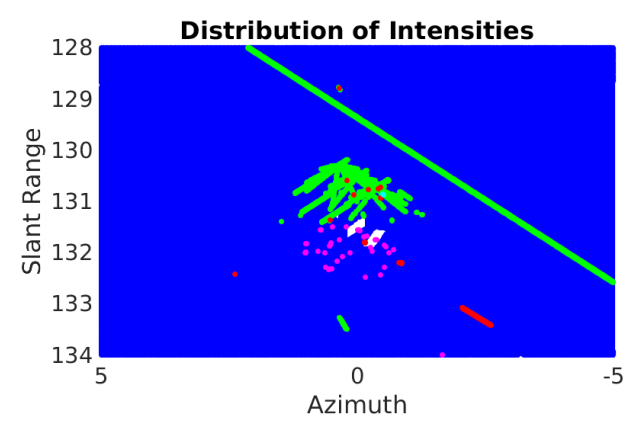

(c) Aspect angle: $45^{\circ}$

Figure 10. Simulated phase center positions for street signs in azimuth and range; signal incidence angle: $40^{\circ}$; blue: single reflection, green: double reflection, red: triple reflection, magenta: fourfold reflection, cyan: fivefold reflection, white: shadow. Unit on axes: meter.

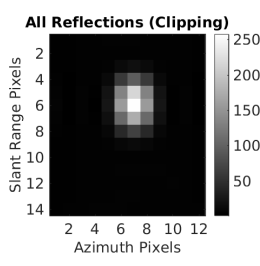

(a) Aspect angle: $15^{\circ}$

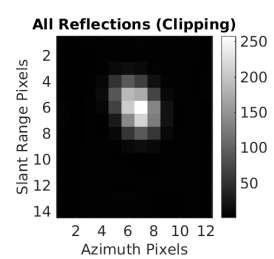

(b) Aspect angle: $30^{\circ}$

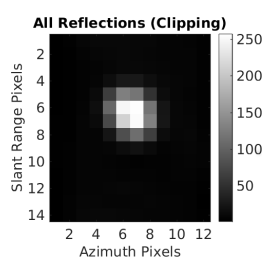

(c) Aspect angle: $45^{\circ}$
Figure 11. Simulated SAR images for street signs (spotlight mode) with an signal incidence angle of $40^{\circ}$.

are expected to be more prominent for the case of horizontal signal polarization (i.e. $\mathrm{HH}$ imaging).

\section{CONCLUSION}

The presented simulation case study indicates that light poles can serve as stable targets in case of orthogonal or near-orthogonal angles between the light pole and the ground beneath. Sloped streets lead to a spatial spread of the related signal phase centers 


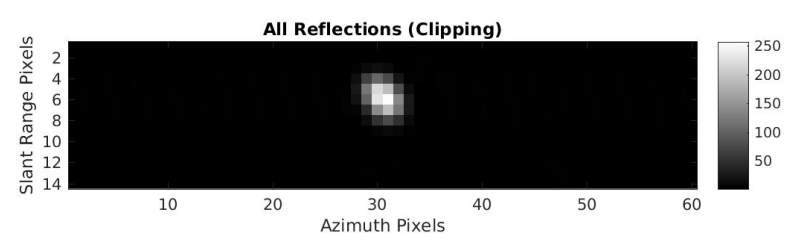

(a) Aspect angle: $15^{\circ}$

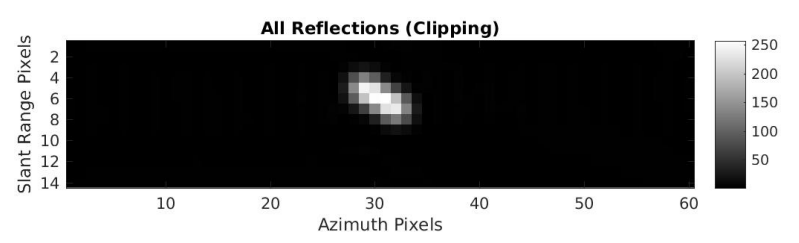

(b) Aspect angle: $30^{\circ}$

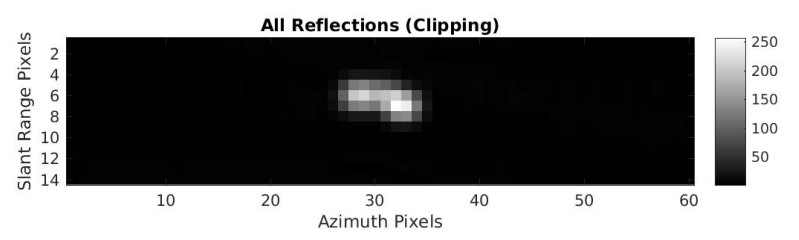

(c) Aspect angle: $45^{\circ}$

Figure 12. Simulated SAR images for street signs (staring spotlight mode) with an signal incidence angle of $40^{\circ}$.

and, hence, do not contribute to dominant image signatures. The simulation exemplifies that dominant signal responses may be composed by small phase-center subgroups - located in compact regions - which are merged to one point signature in the related SAR image. The resulting point target (weighted sum of signal components) indicates a spatially compact nature at the intersection between light post and ground surface (variation with aspect depending on light post diameter), which is promising, e. g., in the context of traffic applications.

With regard to StereoSAR applications, where the 2-D localization information from multiple SAR images with different viewing angle is merged to get 3-D coordinates, a stable phase center position over the entire range of viewing angles in the underlying SAR images is a core assumption. However, the case study showed that with varying aspect angle there is a systematic shifts due to the impact of the light pole diameter. The reflecting surface is moving around the pole with aspect. In case of conic poles also the diameter change with height has to be considered. In order to avoid that the attainable accuracy of acquired 3-D coordinates is ruled by the pole diameter, the choice of SAR images with different elevation angles but similar aspect angles is preferable.

Groups of nearby street signs are merged to point signatures for the high resolution TerraSAR-X imaging modes. The linear arrangement of the street signs (case study: $0.5 \mathrm{~m}$ spacing between 3 street signs in a row) leads to smearing of the corresponding point signature for side views. Thus, the width of the point signature provides a suitable distinctive criterion when (e. g. in case of the StereoSAR application in view) only point signature from single objects shall be processed while superposed signatures from nearby objects shall be excluded. Beyond that, only the object shadow areas provides a further hint to several nearby street signs.

Based on the case study, where the focus was on simulation flexibility, the next scene setting could be adapted to a real scenario with known target, road and pavement positions / orientations and given TerraSAR-X data.

\section{REFERENCES}

Auer, S., 2011. 3D Synthetic Aperture Radar Simulation for Interpreting Complex Urban Reflection Scenarios. $\mathrm{PhD}$ thesis, Technische Universität München.

Auer, S. and Gernhardt, S., 2014. Linear signatures in urban SAR images - partly misinterpreted? IEEE Geosci. Rem. Sens. Lett. (10), pp. 1762-1766.

Auer, S., Bamler, R. and Reinartz, P., 2016. RaySAR - 3D SAR simulator: Now open source. In: Proceedings of IGARSS conference, pp. 6730-6733.

Balss, U., Gisinger, C., Cong, X. Y., Brcic, R., Hackel, S. and Eineder, M., 2014. Precise measurements of the absolute localization accuracy of TerraSAR-X on the base of far-distributed test sites. In: Proceedings of the European Conference on Synthetic Aperture Radar (EUSAR), pp. 993-996.

Balss, U., Runge, H., Suchandt, S. and Cong, X. Y., 2016. Automated extraction of 3-D ground control points from SAR images - an upcoming novel data product. In: Proceedings of IGARSS conference, pp. 5023-5026.

Chen, P. H. and Dowman, I. J., 2001. A weighted least squares solution for space intersection of spaceborne Stereo SAR data. IEEE Trans. Geosci. Remote Sens. 39(2), pp. 233-240.

Eineder, M., Minet, C., Steigenberger, P., Cong, X. Y. and Fritz, T., 2011. Imaging geodesy - toward centimeter-level ranging accuracy with TerraSAR-X. IEEE Trans. Geosci. Remote Sens. 49(2), pp. 661-671.

Fratarcangeli, F., Nascetti, A., Capaldo, P., Mazzoni, A. and Crespi, M., 2016. Centimeter COSMO-SkyMed range measurements for monotoring ground displacements. Int. Arch. Photogramm. Remote Sens. Spatial Inf. Sci. (XLI-B7), pp. 815-820.

Gernhardt, S., Auer, S. and Eder, K., 2014. Persistent scatterers at building facades - evaluation of appearance and localization accuracy. ISPRS J. Photogramm. Remote Sens. 100, pp. 92-105.

Gisinger, C., Balss, U., Pail, R., Zhu, X. X., Montaseri, S., Gernhardt, S. and Eineder, M., 2015. Precise three-dimensional stereo localization of corner reflectors and persistent scatterers with TerraSAR-X. IEEE Trans. Geosci. Remote Sens. 53(4), pp. 17821802.

POV-Ray, 2017. POV-Ray. Persistence of Vision Raytracer Propriety Limited, www.povray.org [checked: 21.03.2017].

Runge, H., Balss, U., Suchandt, S., Klarner, R. and Cong, X. Y., 2016. DriveMark - generation of high resolution road maps with radar satellites. In: Proceedings of 11th ITS European Congress, pp. 1-6.

Sansosti, E., 2004. A simple and exact solution for the interferometric and Stereo SAR geolocation problem. IEEE Trans. Geosci. Remote Sens. 42(8), pp. 1625-1634.

Schubert, A., Jehle, M., Small, D. and Meier, E., 2011. Mitigation of atmospheric perturbations and solid earth movements in a TerraSAR-X time-series. Journal of Geodesy 86, pp. 257-270.

Schubert, A., Small, D., Jehle, M. and Meier, E., 2012. COSMOSkyMed, TerraSAR-X, and Radarsat-2 geolocation accuracy after compensation for earth-system effects. In: Proceedings of IGARSS conference, pp. 3301-3304. 